\title{
Characterization of interaction between electric vehicles and smart grid
}

\author{
ZHANG Yan ${ }^{2, *}$, HE Yongxiu², SU Fengyu², WANG Xiaoqing ${ }^{2}$ and ZHANG Diling ${ }^{1}$ \\ ${ }^{1}$ Beijing Key Laboratory of Demand Side Multi-Energy Carriers Optimization and Interaction Technique, Beijing Haidian District 100085, \\ China \\ ${ }^{2}$ North China Electric Power University, Beijing Changping District 102206, China
}

\begin{abstract}
With the development of smart distribution technology in the future, electric vehicle users can not only charge reasonably based on peak-valley price, they can also discharge electricity into the power grid to realize their economic benefit when it's necessary and thus promote peak load shifting. According to the characteristic that future electric vehicles can discharge, this paper studies the interactive characteristics between electric vehicles and smart grid. In this paper, the example shows that the charging and discharging behaviour of EV users will bring significant fluctuation effect to the power grid load, and the reasonable TOU strategy can stimulate EV users to conduct reasonable charging and discharging, so as to smooth the peak and valley difference of some power grid.
\end{abstract}

\section{Introduction}

In the future, with the development of smart power technology, the coupling and interaction characteristics between the load end and the grid will become more and more significant ${ }^{[1]}$. For electric vehicles, they can carry out the real time electric energy exchange with the grid based on V2G (Vehicle-to-Grid) technology. This technology can use the price signal to guide users to charge and discharge so that users can realize the target of optimizing the power consumption, and the power grid can realize the economic dispatch and alleviate the pressure in peak periods ${ }^{[2-4]}$. At present, many scholars have done relevant research, such as discussing the benefit of different charging modes and combining the EVs with energy storage technologies ${ }^{[5]}$, but the research which integrates electric vehicle and real-time electricity price is less. Balijepalli et al. analysed the benefits of demand response and reviewed the correlational research on it, including research on EVs and this study has referential value for our research to a certain extent. This paper analyses the interaction effect of electric vehicles and grid based on the real time electricity price under different scenarios.

\section{Analysis on characterization of interaction of electric vehicle based on V2G technology}

V2G (Vehicle-to-Grid) technology is a new type of grid technology, which can realize the reverse charging of electric vehicles to the grid. Electric vehicles with V2G can not only serve as consumers of electricity, but also serve as power sources to provide power to the power grid, so as to realize two-way interaction and electrical energy exchange between electric steam and the power grid. Charging of electric vehicles has a strong randomness in time distribution. Under the situation of widespread access of electric vehicles, it is likely to have a certain negative impact on operation management, scheduling planning and other aspects of power grid. This paper takes electric private cars as an example to analyse their electricity characteristics and load curve.

\subsection{Initial charging time of electric vehicle}

Assuming that the travel time, daily mileage and other data of private electric vehicles are the same as those of fuel vehicles, and referring to the results of the National Household Travel Survey (NHTS) conducted by the US Department of Transportation in all 50 states, the most probable likelihood estimation method is adopted to approximate the normal distribution at the beginning of charging for private electric vehicles. Its probability density function is shown in the following equatio ${ }^{[1]} \mathrm{n}$.

$$
f_{s}(t)= \begin{cases}\frac{1}{\sigma_{s} \sqrt{2 \pi}} e^{-\frac{\left(t-\mu_{s}\right)^{2}}{2 \sigma_{s}^{2}}} & \mu_{s}-12<t \leq 24 \\ \frac{1}{\sigma_{s} \sqrt{2 \pi}} e^{-\frac{\left(t+24-\mu_{s}\right)^{2}}{2 \sigma_{s}^{2}}} & 0<t \leq \mu_{s}-12\end{cases}
$$

Where, $t$ is the charging start time of EV,and $\mu_{s}=17.6$, $\sigma_{s}=3.6$

\subsection{Battery capacity and initial charge}

In this paper, it is assumed that the battery capacity of

* Corresponding author: hsezzhangyan@163.com 
electric private cars is gamma distribution. Some battery parameters of electric private cars are shown in Table 1:

Table1. Battery parameters of electric private car

\begin{tabular}{ccccc}
\hline category & parameter $\alpha$ & $\begin{array}{c}\text { parameter } \\
\beta\end{array}$ & $\begin{array}{c}\text { battery } \\
\text { capacity } \\
/ \mathrm{kWh}\end{array}$ & $\begin{array}{c}\text { maximum } \\
\text { capacity for } \\
\text { quick } \\
\text { charging } \\
/ \mathrm{kWh}\end{array}$ \\
\hline parameter & 10.8 & 0.8 & 15 & 5 \\
\hline
\end{tabular}

Note: The maximum capacity of fast charging indicates that fast charging is possible only if the capacity is below this set value

The distribution of battery capacity of electric private cars is shown in Equations (2) (3) :

$$
\begin{aligned}
& f(\text { cap } ; \alpha, \beta)=\frac{1}{\beta^{\alpha} \Gamma(\alpha)} \operatorname{cap}^{\alpha-1} e^{-\frac{c a p}{\beta}}(\alpha, \beta>0) \\
& \Gamma(\alpha)=\int_{0}^{+\infty} x^{\alpha-1} e^{-x} d x
\end{aligned}
$$

Assuming that the decrease of battery charge is in direct proportion to the driving distance of the electric vehicle, the real-time charge $S O C_{t}$ of the electric vehicle at time $\mathrm{t}$ is expressed as:

$$
S O C_{t}=\eta \times S O C_{t-1} \times(1-D / \text { Ran })
$$

Where, $\eta$ represents the energy conversion coefficient, which is used to correct the energy loss caused by the acceleration and braking of electric vehicles, and the value range is $[0.9,1.0] ; \quad S O C_{t-1}$ Represents the charge capacity of the electric vehicle at the time point $t-1 ; \mathrm{D}$ represents the distance travelled between time periods $\mathrm{T}-1$ and $\mathrm{T} ; \mathrm{Ran}$ represents the maximum mileage; $S O C_{0}$ represents the battery charge at the first departure time of that day. Assuming that each charge is full, the battery charge at the departure time is 1 .

\subsection{Charging power}

In the previous EV models, ev charging power was treated as a constant value for the sake of simple calculation. However, in the actual process, the power change curve of lithium battery charging process will also change due to different charging ratio and initial charge. The specific change rule is shown in Figure $1^{[1]}$. It can be seen from the figure that at the beginning of charging, the charging power increases gradually, and then decreases gradually after reaching the peak value.

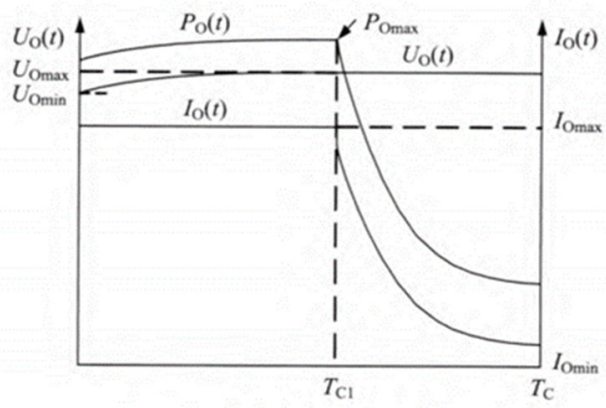

Fig1. Power curve of lithium battery charging

To simplify the analysis, this paper takes the constant current stage as a linear function and the constant voltage stage as an exponential function to obtain the function model of power and time as follows:

$$
P_{O}(t)=\left\{\begin{array}{lc}
I_{O \text { max }} U_{O}(t) & 0 \leq t<T_{C 1} \\
U_{O \text { max }} I_{O}(t) & T_{C 1} \leq t<T_{C}
\end{array}\right.
$$

Set $U_{O \text { min }}=k \times U_{O \max }$, he formula can be deformed as :

$$
P_{O}(t)=\left\{\begin{array}{cc}
U_{O \max } I_{O \max }\left[(1-k) \frac{t}{T_{C 1}}+k\right] & 0 \leq t<T_{C 1} \\
U_{O \max } I_{O \max } \mathrm{e}^{-a\left(t-T_{C}\right)} & T_{C 1} \leq t<T_{C}
\end{array}\right.
$$

\section{Description of interactive characteristics of electric vehicles under time-of-use price}

With the implementation of time-of-use tariff policy, EV users will choose charging time according to the different electricity price, and try to charge when the electricity price is low. Charging decisions made by users at different electricity price levels in different time periods can be described by the demand price elasticity, that is, the ratio between the rate of change of EV charging load and the rate of change of price:

$$
\begin{gathered}
\varepsilon_{u v}=\frac{\Delta Q_{u} / Q_{u}^{(0)}}{\Delta \rho_{v} / \rho_{v}^{(0)}} \\
\Delta Q_{u}=Q_{u}-Q_{u}^{(0)} \\
\Delta \rho_{u}=\rho_{u}-\rho_{u}^{(0)}
\end{gathered}
$$

Where, $Q_{u}^{(0)}$ and $Q_{u}$ are respectively charging loads in $u$ period before and after the implementation of TOU, $\rho_{v}^{(0)}$ and $\rho_{v}$ are respectively the time price before and after the implementation of TOU . When $T_{u}=T_{v}, \varepsilon_{u v}$ is the self-elasticity coefficient $\left(\varepsilon_{u v}<0\right)$, representing the charging demand elasticity of the current period; when $T_{u} \neq T_{v}, \quad \varepsilon_{u v}$ is the cross elasticity coefficient $\left(\varepsilon_{u v}>0\right)$, representing the charging demand elasticity of the electricity price in time period $T_{u}$ to time period $T_{v}$.

After the implementation of TOU, as rational consumers, users can transfer the charging load from the period of high electricity price to the period of low electricity price by flexible charging arrangement (adjustable charging time and power) without changing the charging demand and charging position, thus reducing the charging cost.

The charging load in the trough period is

$Q_{o}=Q_{o}^{(0)}-\Delta Q_{o p}+\Delta Q_{p o}-\Delta Q_{o s}+\Delta Q_{s o}+\Delta Q_{v 2 g}$

The charging load in the flat period is $Q_{s}=Q_{s}^{(0)}-\Delta Q_{s p}+\Delta Q_{p s}-\Delta Q_{s o}+\Delta Q_{o s}+\Delta Q_{v 2 g}$

The charging load during peak hours is 
$Q_{p}=Q_{p}^{(0)}-\Delta Q_{p s}+\Delta Q_{s p}-\Delta Q_{p o}+\Delta Q_{o p}-\Delta Q_{v 2 g}$

Where, $Q_{i}^{(0)}$ represents the initial value of the load in each period, in which i represents different time periods (O represents the trough, $\mathrm{S}$ represents the flat segment, $\mathrm{P}$ represents the peak); $\Delta Q_{i j}$ represents the load transferred from period I to period J, $\Delta Q_{v 2 g}$ represents the load transferred by electric vehicles to the grid through V2G technology.

\section{Example analysis}

In this section, the coupling interaction between electric private cars and the power grid is calculated, and the change of power grid load under the scenarios of $40 \%$ discharge, $60 \%$ discharge and $80 \%$ discharge is respectively calculated under the difference of electricity price between high, medium and low peak and valley.

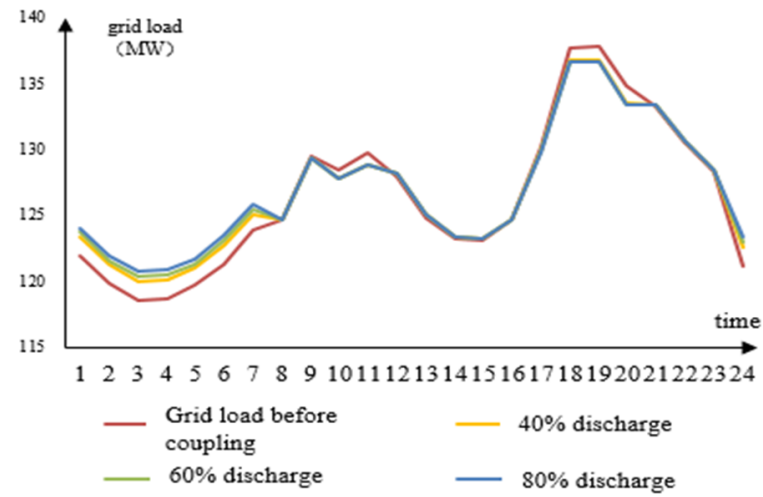

Fig2. Changes of grid load with different discharge ratios of EV under low electricity price

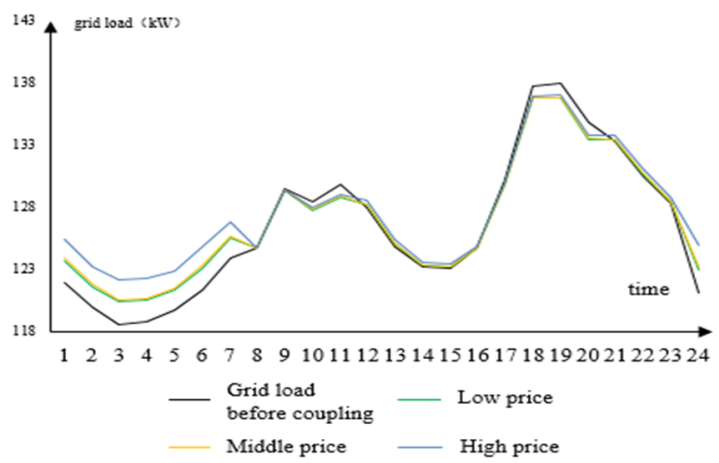

Fig3. Changes of grid load under different electricity prices at $60 \%$ EV discharge

The two figures respectively show the coupling between electric vehicles and the power grid under the condition of low electricity price with different discharge ratio and the change of power grid load under the condition of different electricity price level under the condition of $60 \%$ discharge ratio of electric vehicles. The results show that in the case of $60 \%$ discharge, the higher the electricity price level is, the more obvious the effect of EVs participating in peak load cutting is.

Then the peak-to-valley ratio of grid load is introduced to analyze the coupling effect of EV. Peak-to-valley ratio of power grid load refers to the ratio between the maximum load and the minimum load of power grid, which can reflect the peak-to-valley difference of power grid load in a day. The larger the peak-to-valley ratio is, the more unstable the power grid load is, and the more measures are needed to control the load. The peak-valley ratio of grid load before and after EV interaction is calculated, and the calculation results are shown in Figure 4 below.

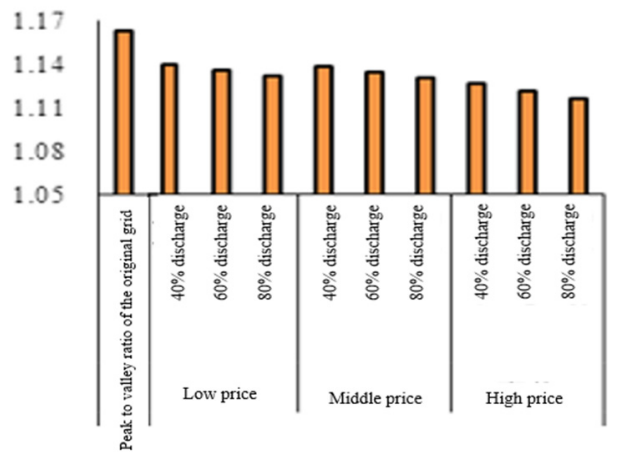

Fig4. Peak-valley ratio of grid load under different scenarios

As can be seen from the figure, before the participation of electric vehicles in coupling, the peak and valley ratio of the power grid is relatively large. Based on the above research, it can be seen that the charging and discharging behaviors of EV users will bring significant fluctuation effect to the power grid load, and a reasonable TOU strategy can motivate EV users to conduct reasonable charging and discharging, so as to smooth the peak and valley difference of part of the power grid.

Therefore, in the future, with the continuous popularization of $\mathrm{V} 2 \mathrm{G}$ technology of electric vehicles, the grid load will be confronted with great fluctuations. In order to suppress the fluctuations caused by large-scale charging and discharging behaviors of electric vehicles on the grid, EV users need to be guided to participate in the coupling interaction with the grid in a reasonable and orderly manner.

\section{Conclusion}

V2G technology effectively realizes the two-way electric energy interaction between electric vehicles and smart grid, which greatly promotes the participation of electric vehicles in the process of demand response. However, the interaction between electric vehicles and the power grid is affected by the peak-valley price difference and the charging and discharging behaviour of electric vehicles. In the future, electric vehicles' participation in the power grid interaction must have a more perfect mechanism to guide the charging and discharging behaviour of electric vehicle users, so as to prevent more fluctuations in the power grid load curve, which is not conducive to the safe and stable operation of the power grid.

\section{Acknowledgments}

This work is supported by Open Fund of Beijing Key Laboratory of Demand Side Multi-Energy Carriers Optimization and Interaction Technique(No.YDB51202001975) 


\section{References}

1. LIU Qing, QI Zhongyi. The Load Forecast Model for Power Grid with the Accessing of Large-scale Electric Vehicles by Considering Spatial Motion Characteristics[J], Modern Electric Power, 2015, 32(1):76-82..

2. Nguyen $\mathrm{H} \mathrm{K}$, Ju B S, Zhu H. Demand side management to reduce Peak-to-Average Ratio using game theory in smart grid. Computer Communications Workshops (INFOCOM WKSHPS), 2012:91-96.

3. Shao S, Pipattanasomporn M, Rahman S. Challenges of PHEV penetration to the residential distribution network. Power \& Energy Society General Meeting, 2009:1-8

4. Li X R, Hui J H, Qian J, et al. Influence of wind power generation on side Load modeling of distribution network. Power system automation, 2009, 33(13):8994.

5. Shang Y L,Sun B,Cui N X, et al. Study on charging Load of large-scale electric vehicles under different charging modes and its influence on power grid. Proceedings of the 33rd China Control Conference. Nanjing :2013. 